\title{
EVALUACIÓN DE LA CIENCIA: TAN NECESARIA COMO PROBLEMÁTICA
}

\section{Science evaluation: necessary and problematic}

\section{Lluís Codina}

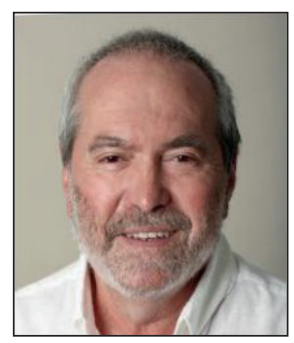

Lluís Codina es profesor de la Universitat Pompeu Fabra (UPF) de Barcelona. Imparte docencia en la Facultad de Comunicación, en los grados de periodismo y de comunicación audiovisual. Es coordinador del Master universitario en comunicación social (Mucs). Forma parte del staff académico e imparte docencia también en los masters universitarios online en documentación digital y en buscadores, de la Barcelona School of Management (UPF). Es coordinador del seminario de investigación del Grupo DigiDoc. Forma parte de la Unidad de Investigación en Periodismo, grupo consolidado reconocido por la Generalitat de Cataluña. Sus últimos libros (en colaboración) son: Calidad en sitios web. Método de análisis general, e-commerce, imágenes, hemerotecas y turismo (Barcelona: EPI Scholar, 2016) y Navigation design and SEO for content-intensive websites: A guide for an efficient digital communication (Oxford: Elsevier-Chandos, 2016).

http://lluiscodina.com

http://orcid.org/0000-0001-7020-1631

Universitat Pompeu Fabra, Departamento de Comunicación Roc Boronat, 138, desp. 53.804, 08018 Barcelona, España luis.codina@upf.edu

\section{Resumen}

Nadie discute la necesidad de que la ciencia sea evaluada, pero su evaluación es motivo de continuas controversias. Se presentan las características de las principales evaluaciones que forman parte del entorno de la investigación, incluyendo las evaluaciones de artículos bajo el sistema peer review y las evaluaciones de trayectorias personales. Se comentan los principales problemas y los retos y posibles soluciones.

\section{Palabras clave}

Ciencia; Academia; Evaluación de la ciencia; Publicación de artículos; Peer review; Tramos de investigación.

\section{Abstract}

No one disputes the need for science to be evaluated, but the evaluation is a source of continued controversy. The characteristics of major evaluations that have been carried out in the scientific research environment are presented in this article, including evaluations of articles under peer review, and evaluations of personal trajectories. The main problems and challenges along with possible solutions are discussed.

\section{Keywords}

Science; Academy; Science evaluation; Papers; Peer review; Personal academic evaluation; Tenure-track.

Codina, Lluís (2016). "Evaluación de la ciencia: tan necesaria como problemática”. El profesional de la información, v. 25, n. 5, pp. 715-719.

\section{Introducción}

Desde un punto de vista teórico, no se sabe de nadie que discuta a estas alturas la necesidad de que la ciencia, para merecer este nombre, debe ser una actividad evaluada. Otra cosa son las reacciones cuando la aplicación de tal evaluación afecta a las carreras académicas y, por tanto, al futuro (y al bolsillo) de los investigadores: léase fracasar en la publicación de artículos, en la obtención de acreditaciones o en alguna clase de reconocimientos que darían derecho a mejores contratos o a aumentos de salario.

Es inevitable, dada la naturaleza humana, que el impacto negativo de algunas evaluaciones en las carreras de los investigadores genere un debate permanente sobre las formas en las que se lleva a cabo, incluso en algunos casos, 
aunque minoritarios, genere un rechazo a la supuesta necesidad de cualquier forma de evaluación. Pero incluso entre aquellos que aceptan los fundamentos de la evaluación, surgen voces críticas ante algunos excesos, así como ante efectos colaterales que causan más daños que beneficio a la ciencia en general.

El fundamento, digamos filosófico, de la ciencia evaluada es el siguiente: la primera virtud de un sistema de pensamiento es la búsqueda de la verdad (John Rawls ${ }^{1}$ ), pero el ser humano no dispone de ningún sistema fiable que le permita saber con absoluta certeza haber alcanzado la verdad absoluta respecto a algo, al menos no si ese algo es más complejo que una simple piedra al sol.

\section{Surgen voces críticas ante algunos exce-} sos de las evaluaciones, así como ante efectos colaterales, que causan más daños que beneficio a la ciencia

A partir de esta simple constatación, la ciencia, al menos desde los últimos dos siglos, exige algo que podemos llamar triangulación para poder estar razonablemente seguros de lo que sus cultivadores, los científicos, intentan ofrecernos.

Esta triangulación es omnipresente: la encontramos en los protocolos de los experimentos, de los análisis y de la obtención de datos empíricos. Pero, y aquí está la clave del tema que nos ocupa en este Observatorio, la vemos también cuando una publicación científica o una autoridad académica debe decidir si un artículo es de calidad o si una trayectoria académica o científica merece ser reconocida.

La triangulación se manifiesta en cada caso de una forma distinta: para decidir sobre la calidad de un artículo, el editor pedirá al menos a dos expertos que opinen sobre el mismo; para que una agencia reconozca una carrera académica, se necesitará un tribunal de expertos que, además, pedirán datos objetivos al investigador que espera ser evaluado, etc.

Una aclaración antes de proseguir: necesariamente tengo como background principal el sistema de evaluación que se sigue en España. Entiendo que no difiere demasiado, en cuanto a su fundamento y filosofía, de los sistemas de evaluación de nuestro entorno, si entendemos por tal el sistema científico-académico europeo, e incluso podríamos decir el sistema atlántico (Europa + Norteamérica). Además, hay cosas en el ámbito de la evaluación de la ciencia, como el sistema peer review, que es prácticamente universal, al menos si no entramos en la casuística de sus formas de aplicación. En cambio, difiere y bastante, en la promoción de carreras académicas, como ya señalaré.

\section{Situación actual}

\section{Empecemos con dos observaciones.}

Primera: si tuviéramos que buscar una forma abstracta de definir cómo funcionan los sistemas de evaluación de la ciencia actuales podríamos decir que consisten en dos tipos de componentes:
1) métricas objetivas;

2) evaluaciones por pares o expertos.

En algunas evaluaciones sólo interviene el segundo de ellos, mientras que en otras consiste en una combinación de ambos. En este último caso, puede suceder que el primer componente, las métricas, tenga un gran peso a expensas del segundo, o al revés.

Segunda: aquello que se somete a evaluación tiene un amplio espectro. Con razón suele decirse que la profesión de investigador es una de las más evaluadas del mundo. Va desde la necesaria para conseguir un título de doctor hasta la evaluación de la trayectoria de un investigador para conseguir un contrato o una mejora del mismo. En medio de todo esto tenemos una auténtica legión de evaluaciones, tanto individuales: solicitudes de beca, de artículos en revistas científicas, de proyectos de investigación, de tramos de investigación, de calidad en la docencia, como colectivas: de acreditación de títulos de grado o de máster, de grupos de investigación, de departamentos, de facultades y ide universidades enteras!

Para simplificar, revisemos someramente dos de las que, por otro lado, causan más polémicas:

- la evaluación de artículos científicos; y

- las evaluaciones de trayectorias académicas.

Son dos cosas muy distintas: los primeros son un pedazo de papel (bueno, acéptenlo como metáfora) y la otra puede ser una vida entera de dedicación. Sin embargo, están muy relacionadas.

Suele decirse que la profesión de investigador es una de las más evaluadas del mundo

La forma más extendida actualmente de evaluar artículos científicos es mediante el conocido sistema peer review. Tengo la obligación de considerar que los lectores de EPI están familiarizados con este sistema. En este punto, solamente señalaré que es un caso puro de evaluación por expertos con todos los problemas de subjetividad que pueden adivinar, pero estos aspectos los dejo para más adelante.

En el otro extremo, tenemos la evaluación de trayectorias investigadoras, es decir, la evaluación de personas de carne y hueso. De éstas, a su vez (al menos en España) tenemos dos:

- la llamada acreditación, para poder conseguir un contrato en una universidad; y

- los tramos de investigación, para ver reconocidos un número de años como investigadores de (razonable) éxito.

En estas últimas, donde se evalúa a seres humanos (y no solamente un trozo de papel) entran en juego tanto los componentes de tipo 1, las métricas, como los de tipo 2, las evaluaciones por expertos. 


\section{Problemas y efectos colaterales}

\subsection{Evaluación de artículos científicos}

El sistema actual de evaluación de artículos científicos, denominado como saben peer review, es uno de los más aclamados y más criticados a la vez. Por un lado, hay un consenso casi total entre editores y agencias de evaluación que solamente son aceptables como aportaciones científicas los artículos publicados en revistas que se someten al peer review. Además, las bases de datos más importantes, como Web of Science y Scopus, sólo aceptan este tipo de revistas en sus repertorios.

Sin embargo, no pasa día sin que algún medio de comunicación e incluso alguna revista de peer review icritiquen el peer review! Ante esto, siempre pienso en el símil de la democracia como sistema de gobierno. Afortunadamente, en esta época casi no está en discusión la legitimidad de la democracia, pero las cosas eran muy diferentes en la primera mitad del siglo XX:

"El peer review es el peor de los sistemas, salvo todos los demás"

En un momento, determinado, harto Winston Churchill de tener que justificar su defensa de la democracia, lanzó una de sus famosas frases: "la democracia es el peor de los sistemas, salvo todos los demás".
De modo que ante la persistencia de las críticas, por un lado, pero la evidencia cotidiana de su necesidad, lo único que se me ocurre es: "el peer review es el peor de los sistemas, salvo todos los demás".

\section{Algunos analistas han calculado en va- rias semanas completas al año el tiempo que cada investigador universitario tiene que dedicar a explicar lo que ha hecho}

Lo que evidencia que el peer review tiene difícil (o imposible) sustitución es que sus críticos, a día de hoy, no proponen alternativas. Son muy conocidos sus defectos, de los que recordaremos algunos brevemente, pero no se sabe de ninguna propuesta viable alternativa (auto publicarse o dejar la elección a decisión del director de cada publicación no sirve).

Hemos dicho que hablaríamos de sus defectos. En mi propia lista de problemas (que tampoco es muy original) señalaría los dos siguientes:

1) en su forma mayoritaria (doble o simple ciego) causa enormes retrasos; y

2) los evaluadores en ocasiones son arbitrarios, amparados en el anonimato que les protege.

Otros señalarían la obviedad de que no es un sistema perfecto: no siempre evita los fraudes, a veces no detecta pla-

IIII SCIMAGO
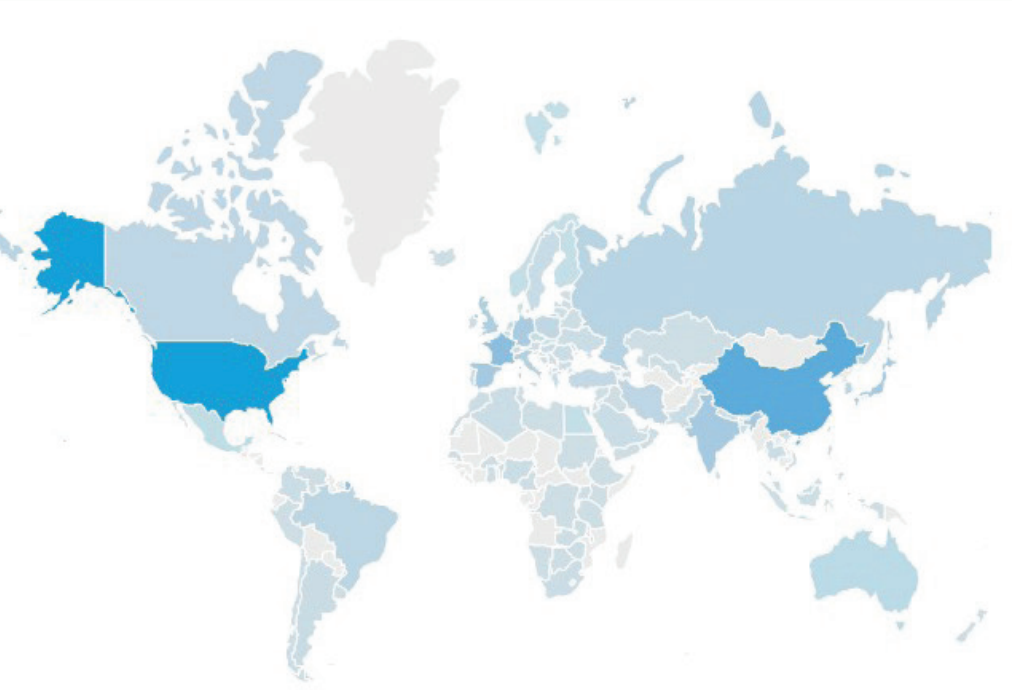
gios, los evaluadores suelen adivinar la identidad de los autores, etc. Dejamos para más adelante, algunas posibles soluciones a los dos primeros problemas, y dejaremos la simple constatación de que no un sistema perfecto como lo que es, una obviedad.

Un problema grave es que nuestras Administraciones tengan la pésima costumbre de solicitar una y otra vez los datos que ya tienen

\subsection{Evaluación de investigadores}

Seguramente, en relación con la evaluación de investigadores, es decir, lo que hemos identificado como evaluación de trayectorias académicas, lo más significativo es su reiteración. Ya hemos avanzado que los investigadores, al menos si pensamos en personal universitario, es uno de los colectivos más evaluados del planeta.

Esto ya, de por sí, es un problema. Algunos analistas han calculado en varias semanas al año de tiempo completo lo que cada investigador universitario tiene que dedicar a explicar lo que ha hecho, dándose la circunstancia de que, cuanto más trabaja y más éxito tiene, mucho peor, porque tiene mucho más trabajo a la hora de hacer las memorias correspondientes.

Otro problema, y algunos creemos que muy grave, es que nuestras Administraciones (hablo del caso español, y por mi circunstancia, también del caso de Cataluña) tienen la pésima costumbre de solicitar una y otra vez lo datos que ya tienen. Personalmente, me he visto rellenando casillas en una evaluación para mi propia universidad indicando cosas como la fecha de entrada en la misma, el nombre de mi departamento o mi tipo de contrato... Sin comentarios...

Por último, para mencionar aún otro problema, en nuestro país se hace una interpretación del progreso en la carrera académica que, a algunos, nos parece aberrante. ¿A qué me refiero? Pues al siguiente y doloroso hecho: una vez que un académico ha acumulado suficientes méritos demostrados por evaluaciones externas e independientes, tiene derecho a intentar consolidar su plaza, tal vez después de más de 10 años de trabajo, todos con buenas evaluaciones y dando lo mejor de sí en su universidad, pero entonces sucede algo asombroso.

La universidad en cuestión no puede revisar su contrato y hacerle uno permanente en virtud de sus logros, como al parecer hacen en las mejores universidades de nuestro entorno (el sistema se conoce como tenure-track), sino que ¡sacan su plaza a concurso público! Es decir, se pone el contador a cero, icomo si toda su trayectoria no existiera y como si las garantías sindicales y constitucionales no existieran para los trabajadores de la universidad! Si nuestro investigador, a pesar de todo, consolida su plaza, entonces pasará a engrosar las estadísticas de endogamia, con lo cual a la ofensa se une el insulto.

\section{Retos y posibles soluciones}

En relación con la evaluación de artículos, algunos confiamos que la posible adopción del sistema de evaluación abierta, u open peer review, podría aliviar algunos de los problemas. La razón es que, en primer lugar, evita el trabajo, con frecuencia tan inútil, de la anonimización. Porque, efectivamente, al menos en la mitad de las ocasiones, según estiman los analistas, los evaluadores adivinan la identidad de los autores.

En segundo lugar, previene algunas malas prácticas por parte de los evaluadores. Como éstos saben que sus evaluaciones se van a hacer públicas y que se van conocer sus identidades, es de suponer que evitarán las críticas superficiales y las arbitrariedades. De paso, ven públicamente reconocida su labor.

En tercer lugar, según algunos editores, si se lleva a cabo en la versión que postulan algunas revistas de ciencias según la cual se unifican los dictámenes de los diferentes evaluadores (tres, típicamente) en un informe consolidado que se hace llegar a los autores, se pueden evitar las segundas rondas.

El sistema de revisión abierta tampoco está exento de problemas, por supuesto. Se trataría de poner en una balanza ventajas e inconvenientes y decidir cuál es el mejor. De momento, hay un nutrido número de revistas en el campo de las ciencias experimentales, especialmente, en medicina y biología, que han adoptado este sistema y no parece que vayan a renunciar a él.

Una vez que un académico ha acumulado y demostrado suficientes méritos e intenta consolidar su plaza sucede algo asombroso: su universidad no puede hacerle un contrato permanente en virtud de sus logros sino que isaca su plaza a concurso público!

Para tranquilidad de los amantes del sistema actual, cabe señalar que la evaluación abierta no varía en cuanto a los pasos esenciales, al menos tal como lo practican las revistas de ciencias. Es decir, existe en primer lugar una decisión editorial, y solamente cuando el artículo es aceptado después de esta primera fase se envía a revisión, normalmente a tres expertos que designa la revista.

Es decir, si se desea, el sistema de revisión abierto puede seguir en todo los pasos del sistema actual, lo que cambia es que tanto autores como evaluadores conocen sus identidades y también, por supuesto, que la revista puede publicar las evaluaciones de los autores así como la versión inicial y final del artículo.

En relación con las evaluaciones de trayectorias académicas sería un logro espectacular que los actores responsables, ya sean agencias de evaluación o rectorados de universidades dejaran de pedir los datos que ya poseen. En segundo lugar, que fueran capaces de utilizar de forma directa el enorme 
caudal de datos públicos que ya existen de los académicos en las bases de datos a las que todas las universidades y agencias tienen acceso.

Sería una auténtica revolución copernicana. El modelo a seguir se podría parecer a lo que hacen las agencias tributarias con nuestros datos. Como la agencia ya tiene casi todos nuestros datos, podemos pedir un borrador de la declaración de cada año y modificar solamente lo que está mal.

Si las agencias de evaluación pusieran en un formulario online los datos que ya tienen, ello representaría una enorme liberación de horas de trabajo de los profesores

Las agencias de evaluación con una cierta inversión inicial en ingeniería, podrían llegar a poner en un formulario online todos los datos que ya tienen procedentes de sus propios archivos y de las bases de datos a las que tienen acceso. De este modo, cuando un profesor tuviera que hacer su enésimo informe, tal vez tendría ya hecha una buena parte del mismo, que solamente tendría que modificar o validar.

Esto redundaría en una enorme liberación de horas de trabajo, que el profesor podría dedicar a labores más productivas vinculadas con su auténtica obligación: la docencia y la investigación (y no rellenar formularios).

Por último, si las universidades españolas adoptaran un sistema internacional como el tenure-track podrían poner el énfasis en el concurso abierto para cubrir las nuevas plazas, y después podrían consolidar a quien lo mereciera, siempre tras una evaluación continuada e independiente. Entonces no haría falta que fingieran que la plaza sale a concurso, porque ya salió en su momento. Por supuesto, también deberían deshacerse de aquellos que no fueran capaces de superar las evaluaciones dentro de un plazo prudencial. Parece que una cosa debería ir con la otra.
En fin, las evaluaciones son necesarias, pero como afectan a nuestras vidas, a nuestros egos y, por supuesto, a nuestro futuro, no podemos esperar que estén exentas ni de críticas ni de debates. Pero lo que siempre podemos esperar es que todo vaya mejorando si, al menos, aceptamos que existen problemas.

\section{Nota}

1. "La justicia es la primera virtud de las instituciones sociales, como la verdad lo es de los sistemas de pensamiento. Por muy elegante y eficiente que sea una teoría, debe ser rechazada o revisada si no es verdad". John Rawls. A theory of justice. Belknap Press, 624 pp. March 31 ${ }^{\text {st }} 2005$, first published Jan. $1^{\text {st }} 1971$. ISBN: 9780674017726

\section{Referencias}

Etkin, Adam (2014). "A new method and metric to evaluate the peer review process of scholarly journals". Publishing research quarterly, v. 30, n. 1, pp. 23-38.

https://doi.org/10.1007/s12109-013-9339-y

Hames, Irene (2007). Peer review and manuscript management in scientific journals: Guidelines for good practice. London: Blackwell, 306 pp. ISBN: 9781405131599

Nicholas, David; Watkinson, Anthony; Volentine, Rachel et al. (2014). "Trust and authority in scholarly communications in the light of the digital transition: Setting the scene for a major study". Learned publishing, v. 27, n. 2, pp. 121-134. https://doi.org/10.1087/20140206

Paolucci, Mario; Grimaldo, Francisco (2014). “Mechanism change in a simulation of peer review: from junk support to elitism". Scientometrics, v. 99, n. 3, pp. 663-688.

https://doi.org/10.1007/s11192-014-1239-1

Vesnic-Alujevic, Lucia (2014). "Peer review and scientific publishing in times of web 2.0". Publishing research quarterly, v. 30, n. 1, pp. 39-49.

https://doi.org/10.1007/s12109-014-9345-8

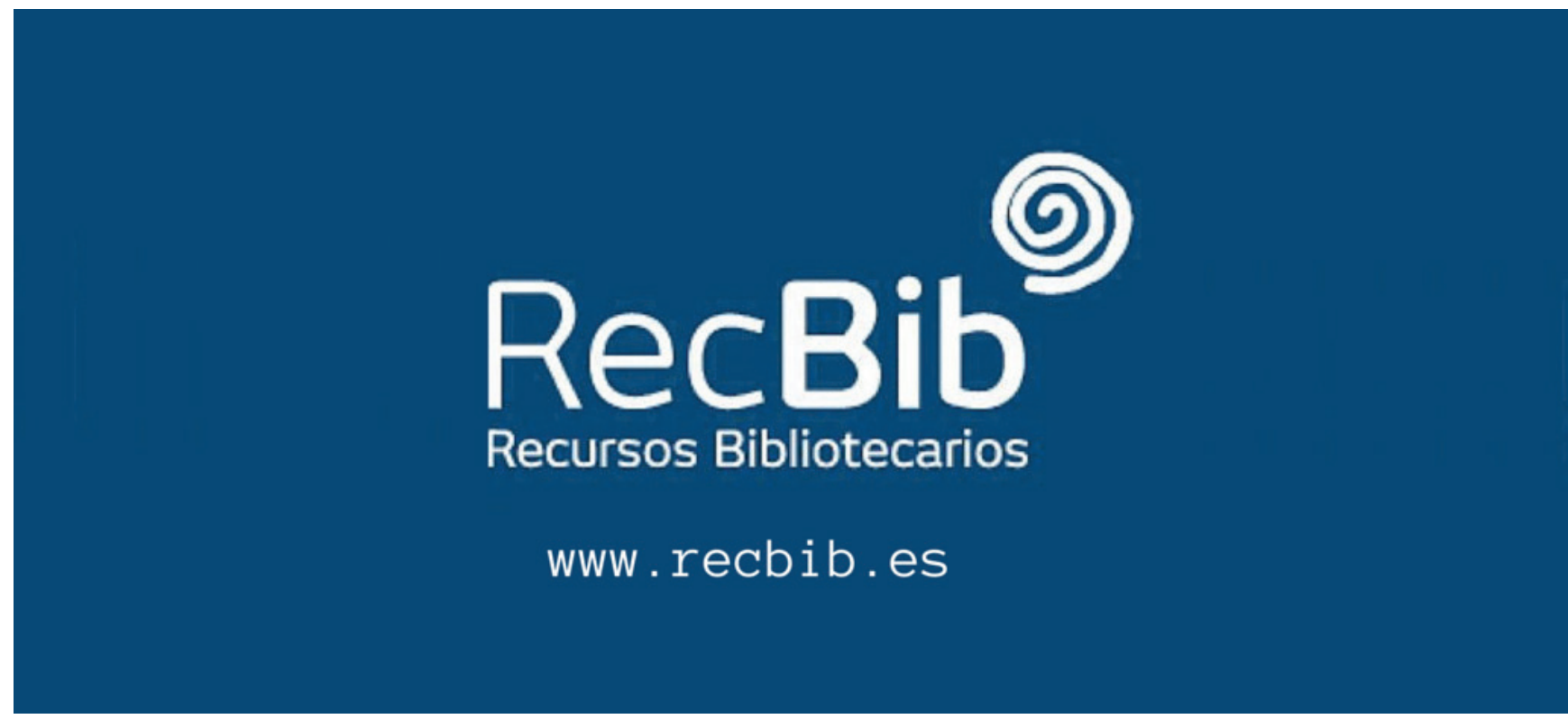




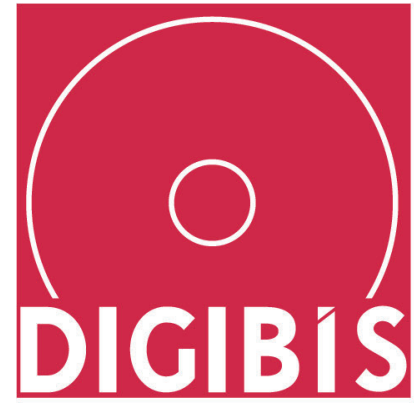

Digitalización enriquecida de fondo antiguo y patrimonial

Software de gestión para Bibliotecas, Archivos y Museos

DIGIBIB $^{\circ}$ DIGIARCH $^{\circ}$ DIGIMUS ${ }^{\circ}$

Recolector OAI-PMH DIGIHUB' de metadatos de diversos proveedores

DIGIBÍS transforma y enriquece las bibliotecas con aplicaciones de gestión digital de nueva generación.

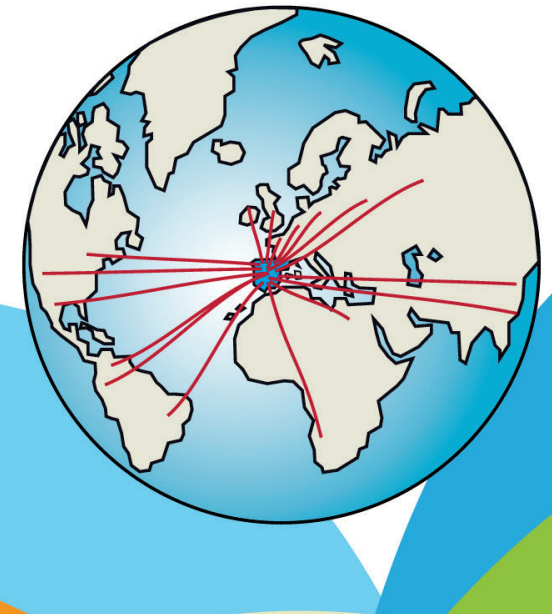

El usuario accede fácilmente a los recursos de la biblioteca desde cualquier lugar, en cualquier momento, con cualquier dispositivo.

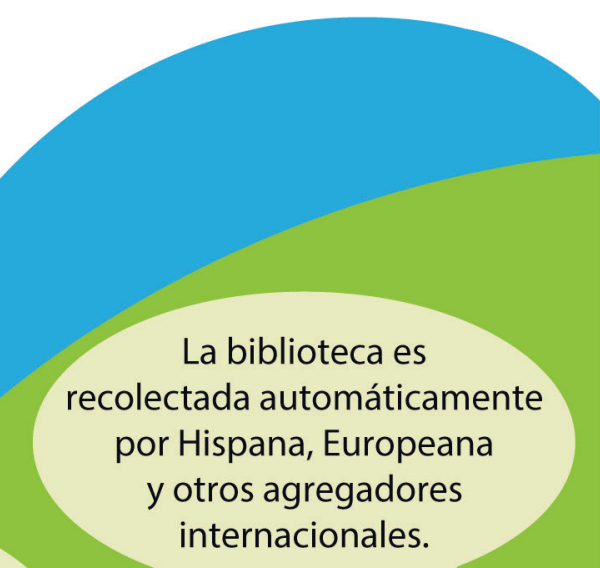

internacionales.

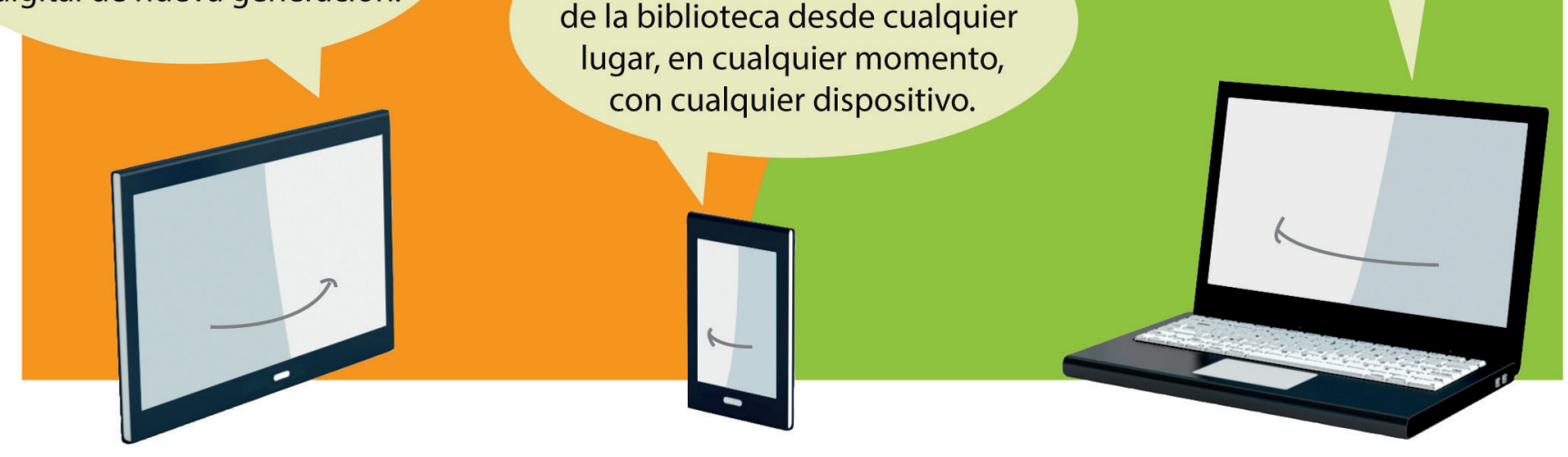

\section{¡Con estándares internacionales para un mundo enlazado y abierto!}

